\title{
Grid-Based Information Aggregation Architecture for Supply Chain Coordination
}

\author{
Ding Fang and Jie Liu \\ Department of Information System and Information Management, Fudan University, \\ Shanghai 200433, P.R. China fangding_fd@126.com liujie@fudan.edu.cn
}

\begin{abstract}
To support supply chain coordination management model's development and popularization, information platform must be able to integrate information resources in the supply chain and make them collaborate dynamically. Grid technologies and infrastructures support the sharing and coordinated use of diverse resources in dynamic, distributed virtual organizations. It's regarded as the promising foundation for constructing supply chain system. This paper proposed grid-based information aggregation architecture for supply chain coordination based on the analysis of the supply chain coordination problem.
\end{abstract}

Keywords: Supply chain coordination, Grid technology, Information aggregation, Enterprise application integration, Inter-enterprise collaboration

\section{INTRODUCTION}

To survive today's business environment, an enterprise needs to consider leveraging resources in an area beyond the boundary of itself. Now the competition of enterprises is indeed the competition of supply chains. After about 20 years' development, both the academic research and enterprise practice indicate that coordination management is the trend of the supply chain management. Currently, the coordination management model for the supply chain information flow and production flow mainly includes VMI (Vendor Managed Inventory), JMI (Jointly Managed Inventory) and CPFR (Collaborative Planning, Forecasting and Replenishment) [1].

It's well known that information technology and the information system (IT/IS) is the enabler of modern business model. Many literatures take IT/IS as one of the key successful factor of the implementation of VMI, JMI and CPFR model. However, because traditional supply chain coordination system has flaws of inflexibility and closeness, they are not sufficient for today's dynamic and adaptive business environment. In order to support the development and popularization of the supply chain coordination management model, information platform must have capability of supporting heterogeneous resources integration and collaboration dynamically. On the other hand, grid technologies are emerging as an infrastructure for next generation business computing, enabling distributed resource management, and large-scale computational problems in science, engineering and commerce [2]. With the

Please use the following format when citing this chapter:

Fang, D.. Liu. J.. 2007, in IFIP International Federation for Information Processing, Volume 254, Research and Practical Issues of Enterprise Information Systems II Volume 1, eds. L. Xu, Tjoa A.. Chaudhry S. (Boston: Springer), pp. 11-18. 
characteristics of openness, heterogeneity, distribution and autonomy, grid technology will be the promising foundation for constructing supply chain coordination system.

The rest of this paper is structured as follows. In the next section, we first introduce the research background from the practice and theory aspect respectively. In section 3 we analyze the supply chain coordination problem. In section 4 we present grid-based information aggregation architecture for supply chain coordination with each major function and key issues explained in detail. We summarize the paper and discuss the future work in the last section.

\section{RESEARCH BACKGROUND}

\section{1) Practice Background}

Without the support of IT/IS, modern business model could not be implemented. The supply chain coordination system's objective is to connect business partners, suppliers, dealers, retailers and even the end customers together, leveraging resource sharing and collaboration of the supply chain, supporting the enterprises to compose dynamic alliance, dealing with the volatile market environment together. Now, many manufacture enterprises and MIS software suppliers both home and abroad have paid much attention to supply chain coordination system. Taking steel and automobile industry as example, Shanghai Baosteel Company, a leading company in china steel industry, has developed collaborative commerce platform with Faw-Volkswagen automotive company and Shanghai General Motors respectively. Chang-an Automobile also developed its collaborative commerce platform with its supplier. Main MIS software suppliers such as SAP, Oracle, i2 have also added the supply chain coordination model to their products.

However, traditional supply chain coordination systems have some critical flaws. The main flaws among them are inflexibility and closeness. These flaws make it difficult for information to share effectively and hard for enterprises to join the collaboration system dynamically. These flaws strictly handicap the development and popularization of coordination management model. For example, the coordination system between Baosteel and Shanghai GM can not automatically integrate the sale plan information, production plan information and stock data of the third logistic company, which can't meet the requirement for the smooth transformation from VMI to JMI and CPER model. Besides, because of the closeness of the system, it will be very hard for other automobile company to join Baosteel's coordination system dynamically. Thus, Baosteel have to develop a new system for every automobile enterprise. The long developing period and high cost (almost 1 million RMB for one system) make the coordination management model only can be implemented with few important partners. To support development and popularization of coordination management model, the supply chain coordination system must have the capability of supporting integration of distribution, dynamic and heterogeneous resources and seamless collaboration of them. 


\section{2) Theory Background}

Grid computing has gained tremendous popularity in the last five years and remains a major topic. The early development of Grid technologies was motivated by the problems of creating scientific resource sharing applications, e.g., collaborative visualization of large scientific data sets, and increasing functionality and availability by coupling scientific instruments and remote computer and archives.

More recently, attempts have been made to open grids to other application fields. Indeed grids appears a promising candidate infrastructure paradigm for managing applications distributed over MAN/WAN and designed to share information and service among users. Thus, aside from computing grids, information grids, knowledge grids and manufacturing grids are now considered with a very high interest.

Ian Foster, who is the head of the distributed systems lab at Argonne National and also an authority in grid field, define grid as a distributed computing infrastructure, coordinated resource sharing and problem solving in dynamic, multi-institutional virtual organizations [3]. In industry community, IBM define grid as any of a variety of levels of virtualization along a continuum, thus, from local area, inside organization, outside organization to global area [4].

Grid has proven itself a viable infrastructure for distributed resource sharing platforms for scientific computing domain. Grid promises to offer solutions to the construction of reliable, scalable, and distributed systems, all of which are very important characteristics of supply chain coordination system [5]. Thus grid technology will be the promising foundation for constructing supply chain coordination system.

\section{SUPPLY CHAIN COORDINATION PROBLEMS}

In VMI model, vendors make the decision of replenishment time, replenishment amount and transportation way by monitoring the stock situation and sales information of the retailer. However, VMI model only transfer risks to the suppliers, the whole supply chain performance is not been improved essentially. Based on the VMI model, two advanced coordination model-JMI and CPFR-has been developed. JMI is a much more detailed extension of VMI but the goals and premise are quite similar. JMI put more emphasis upon managing stock together within partners. JMI included some factors of CPFR, and is considered the transition model form the VMI to CPFR. CPFR's goal is to improve the partnership between trading partners through collaborative processes and shared information. CPFR automates and improves sales forecasting and replenishment between trading partners, enabling participants to share improvements in inventory costs, revenue, and customer service. VICS (American Volunteer Inter-industry Commerce Standards Association) presented CPFR reference operation process [6].

From the CPFR's mission and operation process, we can see that successful CPFR depends on the effective sharing of the information among partners. Advanced Planning and Scheduling (APS) is the plan tool of the supply chain coordination. APS is also a plan method based on the theory of constraints (TOC). In order to make 
feasible coordination plan, enterprises in the supply chain must share customer requirement information, order information, stock information, product data information and production constraint information with their partners. But this information is separated in different system such as PDM, PLM, ERP, CRM, WMS and OA system. Coordination forecasting needs to use information in the CRM and ERP system. Coordination logistics need to use information in the TMS, GPS and GIS system. However, these systems have heterogeneity both in the technology platform and information semantics. For example, these systems were often developed in the different programming environment, such as .Net, J2EE and CORBA, some legacy system even use mainframe environment and COBOL. Meanwhile, for lack of the uniform semantic repertory, information semantics in the supply chain often conflict or wrap with others. All of these are the obstacles of the information aggregation.

\section{GRID-BASED INFORMATION AGGREGATION ARCHITECTURE FOR SUPPLY CHAIN COORDINATION}

Grid architecture identifies fundamental system components, specifies the purpose and function of these components, and indicates how these components interact with one another. Currently there are many researches about the grid architecture [2-3, 7 8]. In this paper we divided these architectures into two categories: general grid architectures and application grid architecture. We will introduce them respectively in the following.

General grid architectures are not limit in certain application field. They only present instructive, abstract architecture framework and could be applied to other application grid, such as computation grid, information grid, manufacturing grid and knowledge grid. Currently there are two important general grid architecture, one is the five-level hourglass framework [2] provided by Foster in 2001, the other is OGSA (open grid service architecture) [3] also provided by Foster etc.

The five-level hourglass framework is a profound structure, which is protocolcentered, and it emphasizes the importance of service, API and SDK. However, its emphasis is not on concrete definition of protocol, but on qualitative description. The five-level framework separates the operation, management and use of sharing resource to five layers by the components and distance of sharing resource. The five layers are fabric layer, connectivity layer, resource layer, collective layer and application layer from the bottom up.

Grid technologies are evolving toward an Open Grid Services Architecture (OGSA) in which a Grid provides an extensible set of services that virtual organizations can aggregate in various ways. Building on concepts and technologies from both the Grid and Web services communities, OGSA defines uniform exposed service semantics (the Grid service); defines standard mechanisms for creating, naming, and discovering transient Grid service instances; provides location transparency and multiple protocol bindings for service instances; and supports integration with underlying native platform facilities. The open source Globus Toolkit described in the "OGSA and the Globus Toolkit" sidebar has emerged as a de facto 
standard for construction of Grid systems. Based on OGSA, Chunming $\mathrm{Hu}$, Jinpeng Huai and Hailong Sun proposed a web service-based grid architecture, which points out how to apply web service into the grid system construction [7]. J. Luo, L. Xu and J. P. Jamont proposed architecture of agent grid, which is an intelligent platform that enables the agents to interact with one another to form dynamic services on the Grid [8].

Application grid architecture could be designed on the base of the domain problems' properties in combination with general grid architecture. For example, Yanli He, Haicheng Yang and Weiping He proposed a manufacturing grid architecture [9]; Mario Cannataro and Domenico Talia proposed knowledge grid architecture [10]. Jin Zhang and Dongyuan Yang proposed logistic information grid architecture [11]. All of these grid architectures are based on the layer structure provided by the general grid architecture.

Since supply chain coordination grid's objective is to solve the integration and collaborative problem of the heterogeneous information resources and application resources in the supply chain, it's an information grid in essence. This paper proposed grid-based supply chain coordination system architecture based on the analysis of the supply chain coordination problems, which is illustrated in figure 1 . The follows will explain each layer's function and key problems in detail.

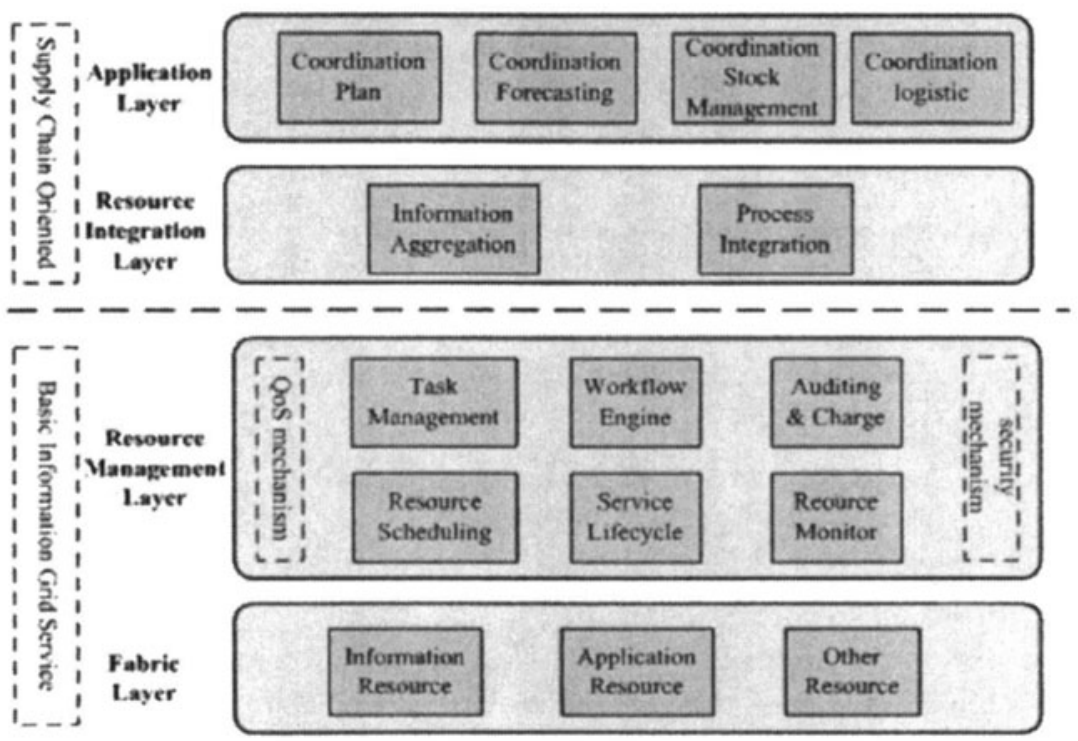

Figure 1. Grid-based Supply Chain Coordination System Architecture

\subsection{Fabric Layer}

This layer provides a set of tools and APIs controlling local resources. In the computation grid, resource means CPU, band width and storage. In the Information 
grid and knowledge grid resource mainly means information and application resource. In the manufacturing grid resource includes equipment and instrument. Like information grid, resource in the grid-based supply chain coordination system includes data resource, meta-data resource, ontology resource and application resource. Data resource covers one enterprise's product technology, customer requirement, stock, order, finance, production and product constraint information. Application resource is the software and agent that contains management science model (production and plan model, forecasting model, logistic model, etc.) and other computation function (such as data mining).

\subsection{Resource Management Layer}

Resource management layer provides the running environment of the resource. Grid services standard provide a standard programming model, which makes it possible for resource to be published, discovered and invoked. The web service protocols cluster provides open, scalable and standard foundation for the implementation and interoperation of resources. For example, WSDL could be used to describe services; UDDI and WS-Inspection could be used to implement service discover and dynamic binding; SOAP could be used to implement service invoking; WS-Security could be used in the security mechanism; BPEL4WS could be used in the service orchestration and workflow management. Based on these protocols, service management and interoperation model could be built.

\subsection{Resources Integration Layer}

In this layer, abstract resource is seamlessly integrated together to provide service to upper layers. According to the dependency degree to the information, resource aggregation could be divided into information aggregation and process integration.

\subsubsection{Information Aggregation Sub-layer}

Information aggregation means sharing understandable data among enterprise in the supply chain. For example, enterprise in the upstream of the supply chain need to know order fulfillment, production capability and production constraint information of downstream enterprises; enterprise in the downstream of the supply chain need to know customer requirement, stock, sales plan information of upstream enterprises.

Information semantic heterogeneity is the main obstacle for the information aggregation in the information grid. Because ontology has good concept structure, support formal definition of the term and support logistic reasoning, it's a promising candidate technology for the information aggregation in information grid. This paper proposed information aggregation architecture based on compound ontology, which is illustrated in figure 2. Data in the grid unit could be abstracted into 3 categories: Meta-data Resource (MR), Data Resource (DR) and Ontology Resource (OR). These resources are represented by grid services and could be invoked by the upper layer 
through the service protocol. The second layer (Resource management layer) is in charge of the management of the lifecycle and availability of these resources. There is an ontology merge \& reasoning module in the information aggregation sub-layer, which could merge local ontology resources into global ontology resource. In combination with the local Meta Data resource and data resource, global aggregation data view could be built.

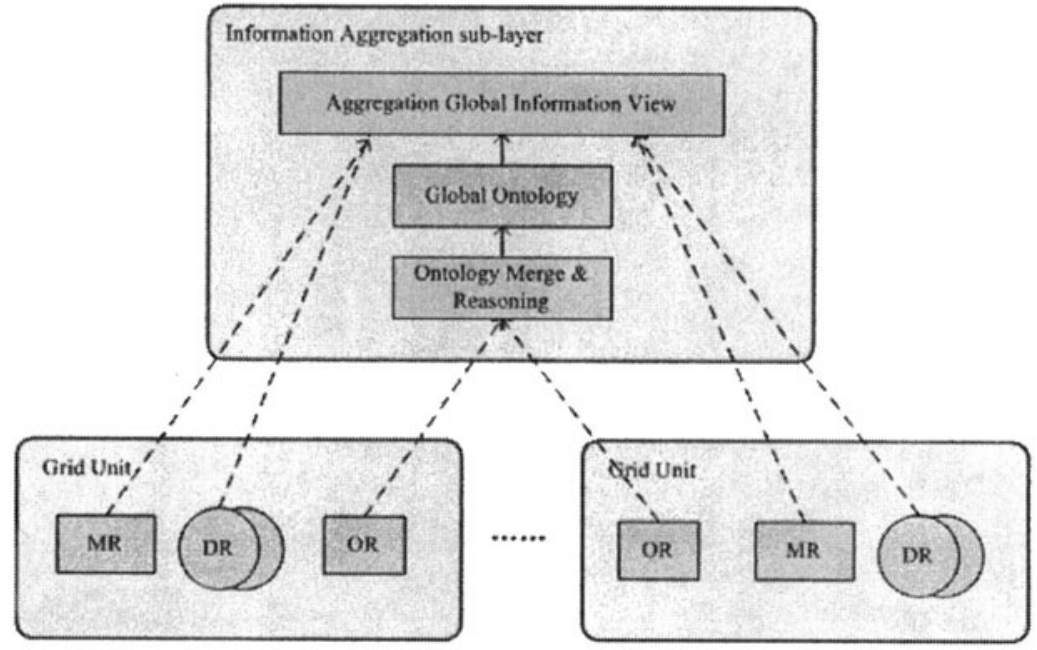

Figure 2. Ontology-Based Information Aggregation Model for Information Grid

\subsubsection{Process Integration Sub-layer}

Information aggregation is the entry-level of the supply chain integration. Process integration's goal is to implement the business process automation. The key issues of the process integration include: business process's description, modeling and decomposition; the orchestration of the business process; the coordination and transaction control of execution of business process.

\subsection{Application Layer}

This is the top layer of the architecture. This layer provides supply chain coordination application service to the user based on the information aggregation and process integration module. Supply chain application services include coordination plan, coordination forecasting, coordination stock management and coordination logistics. The key issues in this layer are the construction and solving method of the management science model. 


\section{SUMMARY AND FUTURE WORK}

In this paper, we have presented a Grid-based information aggregation architectural framework for supply chain coordination. Meta-data, data, ontology and application in the supply chain enterprise are modeled as grid resource and represented by grid services. The components function and every layer's key issues have been described in this paper. The primary focus of our future work in this area will be on the development of more sophisticated ontology based information aggregation module. We are also interested in the business process integration under grid environment and CPFR models in steel and automotive supply chain.

\section{REFERENCES}

1. B. Fugate, F. Sahin, and J.T. Menzter, Supply Chain Management Coordination Mechanisms, Journal of Business Logistics. Volume 27, Number 2, pp.129-161, (2006).

2. I. Foster, C. Kesselman, and J.M. Nick, Physiology of the Grid: An Open Grid Services Architecture for Distributed Systems Integration. Global Grid Forum. http://www.globus.org/research/papers/ogsa.pdf(Accessed July 24, 2006).

3. I. Foster, C. Kesselman, and S. Tuecke, The Anatomy of the Grid: Enabling Scalable Virtual Organizations, The International Journal of High Performance Computing Applications. Volume 15, Number 3, pp.200-222, (2001).

4. B. Jacob, M. Brown, and K. Fukui, Introduction to Grid Computing, IBM Corporation. http://www.redbooks.ibm.com/redbooks/pdfs/sg246778.pdf (Accessed May 5, 2007).

5. J. Jeng, H. Chang, and J. Chung, BPMM: A Grid-Based Architectural Framework For Business Process Meta Management, in Proc. of The 2003 Symposium On Applications And The Internet, eds. S. Helal, Y. Oie, C. Chang, and J. Murai, pp.84-94 (IEEE Computer Society: Washington, DC, USA, 2003).

6. J. Andraski, C. Bremant, and G. Cantwell, Collaborative Planning, Forecasting and Replenishment Version 2.0., Voluntary Interindustry Commerce Standards Association. http://www.vics.org/committees/cpfr/voluntary_v2/CPFR_Tabs_061802.pdf (Accessed May 22, 2007).

7. C. Hu, J. Huai, and H. Sun, Web Service-Based Grid Architecture and Its Supporting Environment, Journal of Software (in Chinese). Volume 15, Number 7, pp.1064-1073, (2004).

8. J. Luo, L. Xu, and J.P. Jamont, Flood Decision Support System on Agent Grid: Method and Implementation, Enterprise Information Systems. Volume 1, Number 1, pp.49-68, (2007).

9. Y. He, H. Yang, and W. He, Framework of Cross-Enterprise Collaborative Manufacturing Based On Grid Theory, Computer Integrated Manufacturing Systems. Volume 11, Number 5, pp.636-641, (2005).

10. M. Cannataro and D. Talia, The Knowledge Grid: Designing, Building, and Implementing an Architecture for Distributed Knowledge Discovery, Communications of the ACM. Volume 46, Number 1, pp.89-93, (2003).

11. J. Zhang and D. Yang, Architecture of Logistics Information Grid, Science and Technology Review. Volume 23, Number 10, pp.48-51, (2005). 